\title{
Impact of microvascular invasion on clinical outcomes after curative-intent resection for intrahepatic cholangiocarcinoma
}

\author{
Liang-Shuo Hu MD, $\mathrm{PhD}^{1}$ | Matthew Weiss $\mathrm{MD}^{2}$ (0) | Irinel Popescu MD ${ }^{3}$ | \\ Hugo P. Marques MD ${ }^{4}$ | Luca Aldrighetti MD ${ }^{5}$ | Shishir K. Maithel MD ${ }^{6}$ | \\ Carlo Pulitano $\mathrm{MD}^{7}$ | Todd W. Bauer MD ${ }^{8}$ | Feng Shen MD
}

George A. Poultsides $M^{10}$ | Oliver Soubrane MD $^{11}$ | Guillaume Martel MD ${ }^{12}$ |

B. Groot Koerkamp MD ${ }^{13} \mid$ Endo Itaru MD ${ }^{14} \mid$ Timothy M. Pawlik MD MPH PhD ${ }^{15}$ (1)

${ }^{1}$ Department of Hepatobiliary Surgery and Institute of Advanced Surgical Technology and Engineering, The First Affiliated Hospital of Xi'an Jiaotong University, Xi'an, China

${ }^{2}$ Department of Surgery, Johns Hopkins Hospital, Baltimore, Maryland

${ }^{3}$ Department of Surgery, Fundeni Clinical Institute, Bucharest, Romania

${ }^{4}$ Department of Surgery, Curry Cabral Hospital, Lisbon, Portugal

${ }^{5}$ Department of Surgery, Ospedale San Raffaele, Milan, Italy

${ }^{6}$ Department of Surgery, Emory University, Atlanta, Georgia

${ }^{7}$ Department of Surgery, Royal Prince Alfred Hospital, University of Sydney, Sydney, Australia

${ }^{8}$ Department of Surgery, University of Virginia, Charlottesville, Virginia

${ }^{9}$ Department of Surgery, Eastern Hepatobiliary Surgery Hospital, Shanghai, China

${ }^{10}$ Department of Surgery, Stanford University, Stanford, California

${ }^{11}$ Department of Hepatobiliopancreatic Surgery and Liver Transplantation, AP-HP, Beaujon Hospital, Clichy, France

${ }^{12}$ Division of General Surgery, Department of Surgery, University of Ottawa, Ottawa, Ontario, Canada

${ }^{13}$ Department of Surgery, Erasmus University Medical Centre, Rotterdam, The Netherlands

${ }^{14}$ Division of Gastroenterological Surgery, Yokohama City University School of Medicine, Yokohama, Japan

${ }^{15}$ Department of Surgery, The Ohio State University Wexner Medical Center, Columbus, Ohio

\section{Correspondence}

Timothy M. Pawlik, MD, MPH, PhD, FACS, FRACS (Hon.), Professor and Chair, Department of Surgery, The Urban Meyer III and Shelley Meyer Chair for Cancer Research, Professor of Surgery, Oncology, Health Services Management and Policy, The Ohio State University, Wexner Medical Center, 395W. 12th Ave., Suite 670, Columbus, Ohio.

Email: tim.pawlik@osumc.edu
Background: Microvascular invasion (MiVI) is a histological feature of intrahepatic cholangiocarcinoma (ICC) that may be associated with biological behavior. We sought to investigate the impact of MiVI on long-term survival of patients undergoing curative-intent resection for ICC.

Methods: A total of 1089 patients undergoing curative-intent resection for ICC were identified. Data on clinicopathological characteristics, disease-free survival (DFS), and overall survival (OS) were compared among patients with no vascular invasion (NoVI), MiVI, and macrovascular invasion (MaVI).

Results: A total of 249 (22.9\%) patients had MiVI, while 149 (13.7\%) patients had MaVI ( \pm MiVI). MiVI was associated with higher incidence of perineural, biliary and adjacent organ invasion, and satellite lesions (all $P<0.01$ ). On multivariable analysis, MiVI was an independent risk factor of DFS (hazard ratios [HR] 1.5; 95\%confidence intervals $[\mathrm{Cl}], 1.3-1.9 ; P<0.001$ ), but not OS (HR 1.1; 95\% $\mathrm{Cl}, 0.9-1.3 ; P=0.379$ ). While MiVI and MaVI patients had similar DFS (median, MiVI 14.0 vs MaVI 12.0 months, HR 0.9; 95\% $\mathrm{Cl}, 0.7-1.2 ; P=0.377$ ), OS was better among MiVI patients 
(median, MiVI 39.0 vs MaVI 21.0 months, HR 0.7; 95\%Cl, 0.5-0.8; $P=0.002$ ). Whereas

nodal metastasis, R1 margin, and postoperative morbidity were associated with early death ( $\leq 18$ months) among patients with MiVI, only nodal metastasis was associated with late (>18 months) prognosis.

Conclusions: Roughly 1 out of 5 patients with resected ICC had MiVI. MiVI was associated with advanced tumor characteristics and a higher risk of tumor recurrence.

KEYWORDS

intrahepatic cholangiocarcinoma (ICC), macrovascular invasion (MaVI), microvascular invasion (MiVI), prognosis

\section{1 | INTRODUCTION}

Intrahepatic cholangiocarcinoma (ICC) is the second most common primary liver tumor after hepatocellular carcinoma (HCC), and its incidence and mortality are increasing worldwide. ${ }^{1,2}$ Surgical resection is currently the only potentially curative treatment option for patients with ICC. ${ }^{3}$ However, long-term survival after curative resection among patients with ICC is disappointing. Specifically, 5-year survival after resection has been reported to be only $20 \%-35 \%$ with over two-thirds of patients experiencing recurrence. $^{4-6}$

The vascular invasion has been identified as one of the strongest risk factors contributing to recurrence and death of patients with ICC after surgery. ${ }^{7,8}$ The 7th and 8th editions of the American Joint Committee on Cancer (AJCC) staging manual incorporate vascular invasion within the T category designation. ${ }^{9}$ Macrovascular invasion ( $\mathrm{MaVI}$ ) is defined as tumor invasion into a major vessel that can be identified by radiological imaging or macroscopic examination. In contrast, the diagnosis of microvascular invasion (MiVI) is largely dependent on histological examination. ${ }^{10}$ Generally, MiVi has been defined as the presence of tumor emboli in a portal radical vein, large capsule vessel, or in a vascular space lined by endothelial cells. ${ }^{10} \mathrm{MiVI}$ has been extensively studied and reported to be a strong indicator of worse outcomes among patients with hepatocellular carcinoma ( $\mathrm{HCC}$ ) after resection or liver transplantation. ${ }^{10-17}$ In fact, MiVI is now recognized as the main cause of $\mathrm{MaVI}$ and intrahepatic metastasis among patients with $\mathrm{HCC}^{18}$ In turn, $\mathrm{HCC}$ with histologically confirmed MiVI have a high risk of recurrence after resection and several studies have suggested that adjuvant therapies, such as transarterial chemotherapy and embolization ${ }^{19,20}$ and sorafenib, ${ }^{21}$ may improve the outcome of these patients. In contrast, the potential impact of MiVI on the long-term outcome of patients with ICC has not been well investigated. As such, the objective of the present study was to define the clinical impact of MiVI on the prognosis of patients after curative-intent resection of ICC using a large, multiinstitutional, international database.

\section{2 | PATIENTS AND METHODS}

\section{1 | Study cohort}

Patients undergoing resection with curative intent for ICC from 1990 to 2015 were collected from a multi-institutional database that included 14 major hepatobiliary centers in the USA, Europe, Australia, and Asia (The Ohio State University, Columbus, Ohio; Johns Hopkins University, Baltimore, Maryland; Emory University, Atlanta, Georgia; Stanford University Medical Center, Stanford, California; University of Virginia Health System, Charlottesville, Virginia and Ottawa General Hospital, Ottawa, Canada, Eastern Hepatobiliary Surgery Hospital, Shanghai, China; Yokohama City University, Yokohama, Japan; Royal Prince Alfred Hospital, Sydney, Australia; Fundeni Clinical Institute, Bucharest, Romania; Beaujon Hospital, Clichy, France; Curry Cabral Hospital, Lisbon, Portugal; San Raffaele Hospital, Milan, Italy; and Erasmus University Medical Centre Rotterdam, Rotterdam, The Netherlands). The diagnosis of ICC was histologically confirmed in all cases. Resection with curative intent was defined as macroscopic removal of all tumors (RO or R1 resection). Patients who underwent palliative or R2 resection, ablation, or intra-arterial therapy and patients with extrahepatic metastasis were excluded. The Institutional Review Boards of each participating institution approved the study.

\section{2 | Data collection and follow-up}

Preoperative variables, including standard demographic, clinicopathological, and tumor-related characteristics, were collected using a standardized data sheet. All resected specimens were subjected to histological analysis and were evaluated for tumor size, number, morphology, differentiation, margin, vascular, biliary and perineural invasion, lymph node status, as well as adjacent organ invasion. MaVI was defined as invasion of the tumor into a major vessel that was identified during the macroscopic examination or radiographic imaging; MiVI was defined as tumor invasion of hepatic veins, portal system, and lymphatic ducts that were visible only on microscopy. ${ }^{10,15}$ 
An $\mathrm{RO}$ resection was defined as a minimum margin length of $>1 \mathrm{~mm}$; the microscopic presence of tumor at the margin or a minimum margin length of $\leq 1 \mathrm{~mm}$ was designated as an R1 resection. Pathologic staging was assigned according to the 8th edition American Joint Committee on Cancer (AJCC) staging guidelines. ${ }^{9}$ Details of the operation were documented and collected, including resection mode, lymphadenectomy, operation time, and intraoperative blood loss.

After discharge, all patients were regularly followed with serum carbohydrate antigen 19-9 (CA19-9), carcinoembryonic antigen (CEA), and imaging studies, including abdominal ultrasonography, computed tomography and/or magnetic resonance imaging (MRI) until the death of the patient or the end of the study. In the present study, the primary endpoints were overall survival (OS) and disease-free survival (DFS). OS was defined as the time duration from the date of initial resection to patient death or the end of the study. DFS was defined as the time duration from the date of initial surgery to tumor recurrence. Recurrence was defined as suspicious imaging findings or biopsyproven tumor. The site of recurrence was categorized as intrahepatic and/or extrahepatic. Treatments of recurrence were tailored according to the tumor burden and general condition of the patient. Curativeintent therapies for recurrence included surgical re-resection, ablation, or combined resection plus ablation.

\section{3 | Statistical analysis}

Continuous variables were expressed as medians with interquartile ranges; student $t$ test or the Mann-Whitney $U$ test were used for statistical analysis as appropriate. Categorical variables were expressed as number and percentages and compared with $\chi^{2}$ test or Fisher's exact test. Survival was analyzed by the life table and Kaplan-Meier method and compared with the logrank test. Factors associated with OS and DFS were identified using univariate and multivariable Cox proportional hazards regression models. Hazard ratios $(\mathrm{HR})$ and $95 \%$ confidence intervals $(\mathrm{Cl})$ were estimated. The variables with a $P$ value less than 0.05 on univariate analysis were included in the multivariable models. A two-tailed $P$ value less than 0.05 was considered statistically significant. Statistical analyses were performed using SPSS version 21.0 (IBM SPSS Inc, Chicago, IL).

\section{3 | RESULTS}

\section{1 | Baseline characteristics}

Among 1,089 patients who underwent curative-intent resection for ICC, 149 (13.7\%) had MaVI with or without MiVI, while 249 (22.9\%) had MiVI on histological examination without evidence of MaVI. The clinicopathological characteristics and surgery details among patients with no vascular invasion ( $\mathrm{NoVI}$ ) vs $\mathrm{MiVI}$ vs $\mathrm{MaVI}$ were compared (Table 1). Although tumor size and number, as well as serum markers (ie Ca19-9 and CEA) were not different among the three groups, tumors with either macro- or MiVI had a higher incidence of perineural, biliary and adjacent organ invasion, as well as the presence of satellite lesions (all $P<0.01$ ). In addition, patients with vascular invasion were more likely to have advanced AJCC T disease, lymph node metastasis, and poor tumor differentiation vs patients with NoVI (all $P<0.05$ ). Although patients with macro- or MiVI were more likely to undergo a major hepatectomy and concomitant lymphadenectomy (both $P<0.01$ ), the incidence of an RO resection was lower among patients with vs without vascular invasion (MaVI 79.2\%, MiVI 80.3\% vs NoVI 92.0\%; $P<0.001$ ). Among patients who had the vascular invasion, patients with MaVI had a higher incidence of adjacent organ invasion (13.4\% vs $5.6 \%$ ), nodal metastasis (37.7\% vs $22.9 \%$ ) compared with patients who had MiVI (all $P<0.05)$. In contrast, tumor size, number, as well as the incidence of the perineural and biliary invasion were equivalent among patients with MaVI vs MiVI. The frequency of adjuvant chemo- and radiotherapy was comparable among patients with $\mathrm{MiVl}$ and $\mathrm{MaVl}$; of note, the use of chemotherapy among these patients was higher than patients without vascular invasion (MiVI 38.3\%, MaVI 39.8\% vs NoVI 24.7\%; $P<0.001$ ).

\subsection{Survival of patients stratified by vascular invasion}

After a median follow-up of 35 (range 3-211) months, a total of 553 (50.8\%) patients had died. In examining the entire cohort, 1-, 3- and 5 -year OS was $78.8 \%, 49.9 \%$, and $38.7 \%$, respectively, while 1-, 3and 5 -year DFS was $57.4 \%, 35.2 \%$ and $19.5 \%$, respectively. Of note, patients with MiVI had a better OS compared with patients who had MaVI (median OS, MiVI 39.0 vs MaVI 21.0 months; HR 0.7; $95 \% \mathrm{Cl}$, 0.5-0.8; $P=0.002$ ), which was comparable with patients who had NoVI (median OS, MiVI 39.0 vs NoVI 45.0 months; HR 1.2; $95 \% \mathrm{Cl}$, 0.9-1.5; $P=0.194$ ) (Figure $1 \mathrm{~A}$ ). In contrast, patients with MiVI had a similar DFS as patients with MaVI (median DFS, MiVI 14.0 vs MaVI 12.0 months, HR 0.9; 95\% Cl, 0.7-1.2; $P=0.377$ ), which was worse than patients with NoVI (median DFS, MiVI 14.0 vs NoVI 21.0 months, HR 1.5; 95\% Cl 1.3-1.9; $P<0.001$ ) (Figure 1B).

On multivariable analysis, after taking into account competing risk factors, $\mathrm{MaVI}$ (HR 1.5; 95\% $\mathrm{Cl}, 1.1-1.9 ; P=0.005)$, rather than MiVI (HR 1.1; 95\%Cl, 0.9-1.3; $P=0.379$ ), was associated with worse long-term survival (Table 2). In contrast, both MaVI (HR 1.4; $95 \% \mathrm{Cl}$, 1.0-1.8; $P=0.022$ ) and MiVI (HR 1.6; 95\% Cl, 1.3-2.0; $P<0.001)$, tumor size, number, and differentiation, as well as lymph node status were correlated with risk of tumor recurrence (Table 3).

\subsection{Recurrence and treatments}

During follow-up, 729 (66.9\%) patients experienced tumor recurrence after surgery. Among patients who recurred, patients with $\mathrm{MaVI}$ or MiVI were more likely to develop extrahepatic recurrence than patients with NoVI (MaVI 42.2\%, MiVI 41.8\% vs NoVI 31.6\%; $P=0.003$ ) (Figure $2 \mathrm{~A}$ ). The recurrence pattern was similar among patients with MiVI vs MaVI (Figure 2A). Among 626 patients who were treated for recurrence, patients with $\mathrm{MiVI}$ were more likely to undergo a subsequent curativeintent treatment compared with patients with MaVI (17.7\% vs $7.0 \%$; $P=0.033)$. The utilization of repeat curative-intent treatments for recurrences was similar among patients with $\mathrm{NoVI}$ and $\mathrm{MiVI}$ who recurred (17.7\% vs $12.1 \% ; \quad P=0.082$ ) (Figure 2B). Perhaps not 
TABLE 1 Clinicopathological characteristics and surgical treatments of patients undergoing curative resection for intrahepatic cholangiocarcinoma stratified by vascular invasion status

\begin{tabular}{|c|c|c|c|c|}
\hline & $\begin{array}{l}\text { No vascular } \\
\text { invasion }(n=691)\end{array}$ & $\begin{array}{l}\text { Microvascular } \\
\text { invasion }(n=249)\end{array}$ & $\begin{array}{l}\text { Macrovascular } \\
\text { invasion }(n=149)\end{array}$ & $P$ value \\
\hline Age, y & $59(50-66)$ & $63(54-71)$ & $62(52-71)$ & $<0.001$ \\
\hline Body mass index, $\mathrm{kg} / \mathrm{m}^{2}$ & $25.3(22.4-28.0)$ & $24.8(22.6-28.4)$ & $24.6(21.2-27.0)$ & 0.183 \\
\hline Carbohydrate antigen $19-9, \mathrm{U} / \mathrm{mL}$ & 43.5 (15.1-180.2) & $60.0(20.0-239.3)$ & $60.9(22.8-382.0)$ & 0.515 \\
\hline Multiple lesions $(\geq 2)$ & $108(15.6 \%)$ & $42(16.9 \%)$ & $22(14.8 \%)$ & 0.412 \\
\hline Perineural invasion & 55 (8.0\%) & 81 (32.5\%) & $63(42.3 \%)$ & $<0.001$ \\
\hline Direct invasion of adjacent organs & $43(6.2 \%)$ & $12(5.6 \%)$ & $20(13.4 \%)$ & $<0.001^{*}$ \\
\hline Biliary invasion & $42(6.1 \%)$ & $51(20.5 \%)$ & 39 (26.2\%) & $<0.001$ \\
\hline T3-4 & 64 (9.3\%) & $58(23.3 \%)$ & $44(29.6 \%)$ & \\
\hline Missing & 49 (7.1\%) & $74(29.7 \%)$ & 44 (29.5\%) & \\
\hline AJCC $N$ status & & & & $<0.001^{*}$ \\
\hline NO & 407 (61.2\%) & $94(42.2 \%)$ & 47 (44.3\%) & \\
\hline N1-2 & 79 (11.9\%) & $51(22.9 \%)$ & $40(37.7 \%)$ & \\
\hline $\mathrm{Nx}$ & $179(26.9 \%)$ & $31(35.0 \%)$ & 19 (17.9\%) & \\
\hline Histological grade & & & & 0.007 \\
\hline Well/ moderately differentiated & $548(79.3 \%)$ & $162(65.1 \%)$ & $83(55.7 \%)$ & \\
\hline Operation time, min & $174(108-254)$ & $310(210-436)$ & $301(180-640)$ & 0.474 \\
\hline Postoperative morbidity & $228(33.0 \%)$ & $106(42.6 \%)$ & $55(36.9 \%)$ & $<0.001$ \\
\hline Adjuvant chemo- and radiotherapy & $171(24.7 \%)$ & 99 (39.8\%) & 57 (38.3\%) & $<0.001$ \\
\hline
\end{tabular}

Abbreviation: AJCC, American Joint Committee on Cancer.

*indicates a $P$ value less than 0.05 when compared between microvascular and macrovascular invasion groups.

surprisingly, median OS after the first recurrence among patients with MaVI (8.0 months) was worse than patients with MiVI (17.0 months) or NoVI (13.0 months) $(P<0.001)$.

\section{4 | Survival of patients with MiVl: Early death versus long-term survival}

In examining OS of patients with MiVI vs MaVI, long-term survival was initially generally similar, yet became divergent beginning roughly around 18 months after surgery (cumulative survival rate at 18 months, MiVI 63.9\% vs MaVI 55.5\%; $P=0.01$ ) (Figure $1 \mathrm{~A}$ ). Overall, 79 patients with MiVI died within 18 months after initial surgery (early death), whereas 118 patients survived longer than
18 months (long-term survival). Patients who died within 18 months after initial surgery were more likely to have multiple tumors (26.6\% vs $13.6 \% ; P=0.054)$, lymph node metastasis $(32.9 \%$ vs $16.0 \% ; P=0.024)$, and an $\mathrm{R} 1$ resection $(31.6 \%$ vs $13.6 \%$; $P=0.003$ ) (Table 4). On multivariable analysis, lymph node metastasis ( $\mathrm{HR} 2.6 ; 95 \% \mathrm{Cl}, 1.4-5.0 ; P=0.004$ ), R1 vs R0 margin (HR 2.4; 95\% Cl, 1.1-5.0; $P=0.025$ ), and postoperative morbidity (HR 1.4; 95\% Cl, 1.0-2.0; $P=0.05$ ) were independently associated with early death among patients with MiVI (Table 5). In contrast, among the 118 patients who survived longer than 18 months after initial surgery, only lymph node metastasis was correlated with late death (after 18 months) (HR 3.3; 95\% Cl, 1.5-7.0; $P=0.002$ ) (Table 6). 
(A)

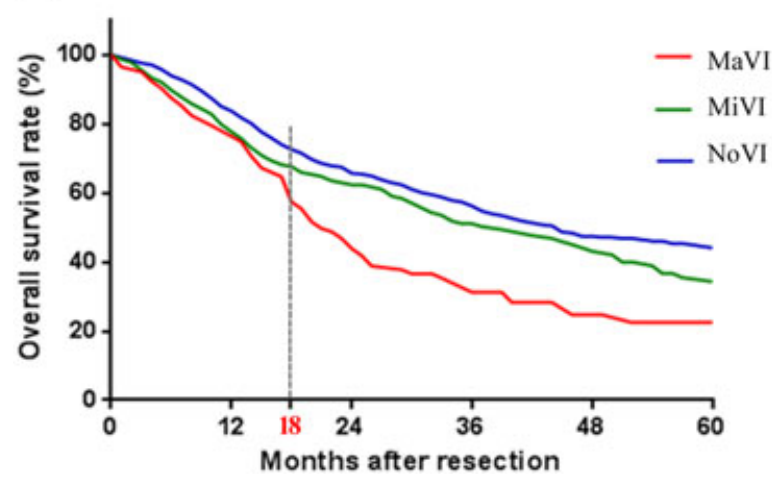

(B)

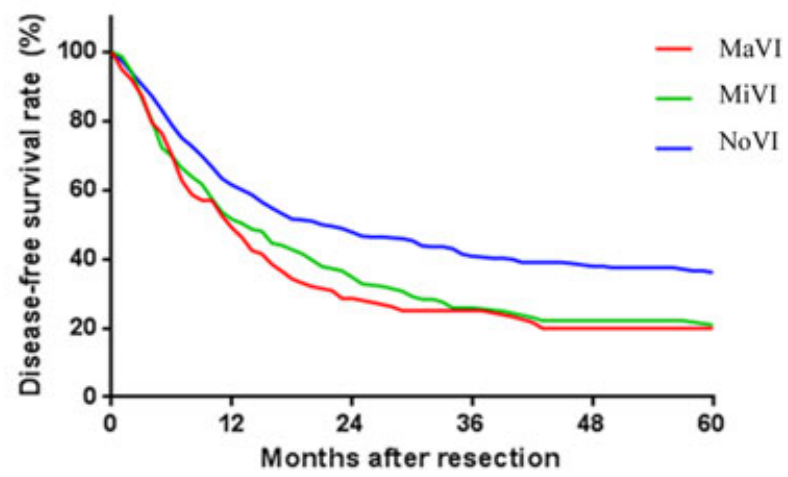

FIGURE 1 Overall (A) and disease-free survival (B) of patients undergoing curative-intent resection for intrahepatic cholangiocarcinoma stratified by vascular invasion status. NoVI, none vascular invasion; MiVI, microvascular invasion; MaVI, macrovascular invasion [Color figure can be viewed at wileyonlinelibrary.com]

TABLE 2 Risk factors for the overall survival of the whole cohort

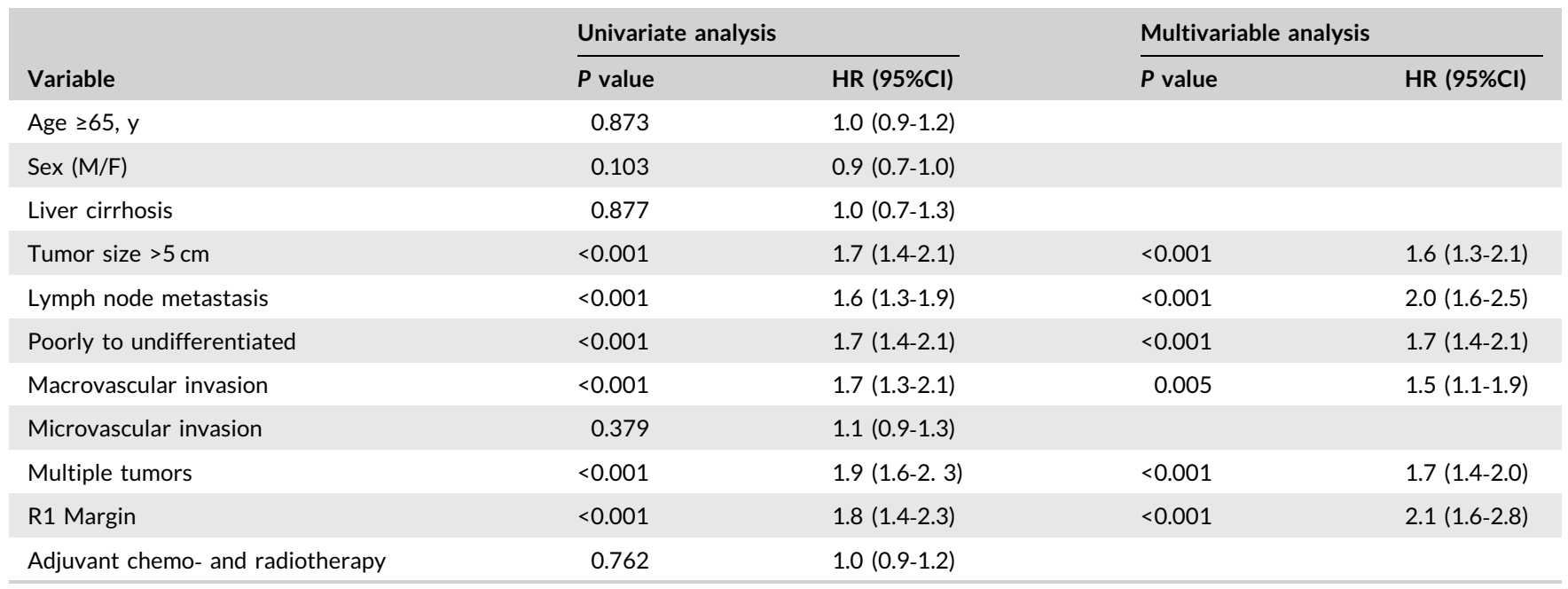

Abbreviations: $\mathrm{Cl}$, confidence intervals; $\mathrm{HR}$, hazard ratios .

TABLE 3 Risk factors for disease-free survival of the whole cohort

\begin{tabular}{|c|c|c|c|c|}
\hline \multirow[b]{2}{*}{ Variable } & \multicolumn{2}{|c|}{ Univariate analysis } & \multicolumn{2}{|c|}{ Multivariable analysis } \\
\hline & $P$ value & HR $(95 \% \mathrm{Cl})$ & $P$ value & HR $(95 \% \mathrm{Cl})$ \\
\hline Age $\geq 65$ y & 0.458 & $0.9(0.8-1.1)$ & & \\
\hline Liver cirrhosis & 0.524 & $0.9(0.7-1.2)$ & & \\
\hline Tumor size $>5 \mathrm{~cm}$ & $<0.001$ & $2.1(1.7-2.5)$ & $<0.001$ & $2.0(1.6-2.5)$ \\
\hline Poorly to undifferentiated & $<0.001$ & $1.5(1.2-1.8)$ & 0.022 & $1.3(1.0-1.6)$ \\
\hline Macrovascular invasion & 0.001 & $1.5(1.2-1.8)$ & 0.022 & $1.4(1.0-1.8)$ \\
\hline Microvascular invasion & 0.002 & $1.4(1.1-1.6)$ & $<0.001$ & $1.6(1.3-2.0)$ \\
\hline Multiple tumors & $<0.001$ & $1.8(1.5-2.2)$ & $<0.001$ & $1.6(1.3-2.5)$ \\
\hline
\end{tabular}

Abbreviations: $\mathrm{Cl}$, confidence intervals; $\mathrm{HR}$, hazard ratios. 
(A)

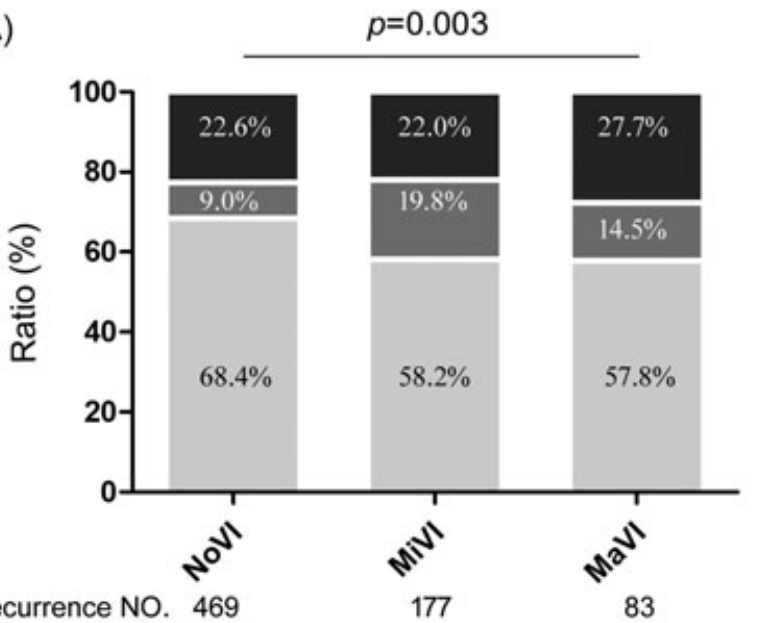

(B)

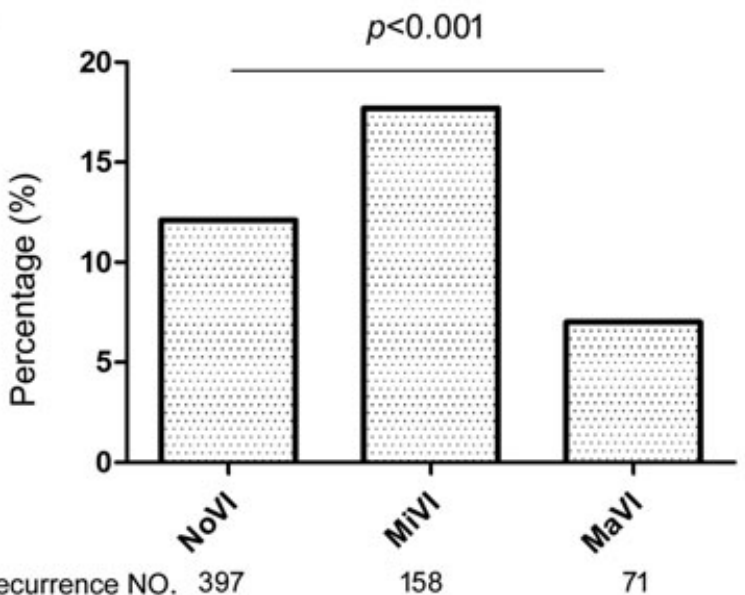

Recurrence NO. 397

\section{4 | DISCUSSION}

The AJCC 8th T classification incorporates vascular invasion as a staging parameter for ICC, yet fails to distinguish the impact of micro- vs macrovascular invasion. ${ }^{9}$ Whether long-term survival of patients undergoing curative-intent surgery for ICC differs relative to micro- vs macro-vascular invasion has been poorly investigated. In the present multi-institutional study, both micro- and macrovascular invasion of ICC were associated with advanced tumor characteristics and stage. Compared with NoVI, the presence of MiVI increased the risk of tumor recurrence, yet was not associated with worse longterm survival after surgery. In contrast, MaVI was a strong indicator of both worse long-term and disease-free survival. The reason for these disparate results was likely done to a higher proportion of MiVI patients being candidates for repeat curative treatments at the time of recurrence compared with $\mathrm{MaVI}$ patients. Of note, early death among patients with MiVI was associated with tumor status (nodal metastasis), surgical technique (R1 margin), and postoperative complications. In contrast, the late death of patients was associated only with biological factors such as the presence of lymph node metastasis.

In the liver, small blood vessels are composed of an inner layer of endothelial cells surrounded by a basal membrane. When tumor
Recurrence site:

Intrahepatic only

- Extrahepatic only

- Intra \& extrahepatic

$\square$ Curative treatment
FIGURE 2 A, Recurrence pattern after curative-intent resection for intrahepatic cholangiocarcinoma among patients with no vascular invasion (NoVI), microvascular invasion (MiVI) or macrovascular invasion (MaVI). B, The proportion of patients receiving curative-intent treatments for recurrence in $\mathrm{NoVI}, \mathrm{MiVI}$ and $\mathrm{MaVI}$ groups

cells in the liver have a sufficiently evolved phenotype, these cells can invade either the portal vein or hepatic vein branches leading to intrahepatic recurrence or systematic metastasis. ${ }^{10} \mathrm{MiVI}$ has been reported to be an independent predictor of tumor recurrence and mortality after resection or transplantation of $\mathrm{HCC}^{10-17}$ In turn, MiVI is commonly utilized in the pathological assessment and prognostic stratification of patients with $\mathrm{HCC}^{10-17}$ Data in the present study demonstrated that $\mathrm{MiVI}$ was an independent risk factor for tumor recurrence, but not long-term survival among patients with ICC. Specifically, patients with only MiVI had the same DFS compared with patients who had MaVI, as well as a comparable recurrence pattern (intrahepatic recurrence: $58.2 \%$ vs $57.8 \%$ ). Interestingly, previous data had suggested that AJCC stage II HCC patients with MiVI had similar outcomes as patients with multiple tumors, implying that MiVI was a strong risk factor for intrahepatic recurrence. ${ }^{13}$ In the present study, patients with ICC and histologically confirmed MiVI or MaVI had a higher incidence of perineural and biliary invasion, satellite lesions, advanced AJCC T stages, nodal metastasis, and poor differentiation. Given the association of vascular invasion with generally advanced disease, patients with MiVI in the pathological liver specimen should be closely surveilled for early detection and possible treatment of any recurrence. To this point, several studies have reported that 
TABLE 4 Clinical and pathological characteristics of early dead ( $\leq 18$ months) versus long-term survived (>18 months) patients with microvascular invasion

\begin{tabular}{|c|c|c|c|}
\hline & $\begin{array}{l}\text { Early } \\
\text { death } \\
(n=79)\end{array}$ & $\begin{array}{l}\text { Long-term } \\
\text { survived } \\
\text { ( } n=118 \text { ) }\end{array}$ & $P$ value \\
\hline Age, years & $63(52-71)$ & $63(56-70)$ & 0.581 \\
\hline Men & 47 (59.5\%) & $56(47.5 \%)$ & 0.127 \\
\hline $\mathrm{BMI}, \mathrm{kg} / \mathrm{m}^{2}$ & $\begin{array}{l}24.7 \\
(22.1-29.2)\end{array}$ & $24.7(22.5-28.1)$ & 0.419 \\
\hline Tumor Size, cm & $7.0(5.0-8.7)$ & $6.5(4.0-9.5)$ & 0.576 \\
\hline Multiple lesions $(\geq 2)$ & $21(26.6 \%)$ & $16(13.6 \%)$ & 0.054 \\
\hline Perineural invasion & 30 (38.0\%) & $36(30.5 \%)$ & 0.323 \\
\hline $\begin{array}{l}\text { Direct invasion of adjacent } \\
\text { organs }\end{array}$ & $4(5.1 \%)$ & $5(4.2 \%)$ & 0.997 \\
\hline Biliary invasion & $16(20.3 \%)$ & $22(18.6 \%)$ & 0.676 \\
\hline Satellite lesions & $29(36.7 \%)$ & $28(23.7 \%)$ & 0.063 \\
\hline AJCC tumour category & & & 0.455 \\
\hline $\mathrm{T} 1-2$ & $44(55.7 \%)$ & $55(46.6 \%)$ & \\
\hline T3-4 & $23(29.1 \%)$ & $23(19.5 \%)$ & \\
\hline Missing & $12(15.2 \%)$ & 40 (33.9\%) & \\
\hline AJCC node category & & & 0.024 \\
\hline NO & $28(35.4 \%)$ & $46(46.0 \%)$ & \\
\hline N1-2 & $26(32.9 \%)$ & $16(16.0 \%)$ & \\
\hline $\mathrm{Nx}$ & $20(25.3 \%)$ & 38 (38.0\%) & \\
\hline Histological grade & & & 0.105 \\
\hline $\begin{array}{l}\text { Well to moderately } \\
\text { differentiated }\end{array}$ & 49 (62.0\%) & 78 (66.1\%) & \\
\hline $\begin{array}{l}\text { Poorly to } \\
\text { undifferentiated }\end{array}$ & 22 (27.8\%) & 18 (15.3\%) & \\
\hline Missing & $8(10.1 \%)$ & $22(18.6 \%)$ & \\
\hline RO resection & $54(68.4 \%)$ & $102(86.4 \%)$ & 0.003 \\
\hline Lymphadenectomy & 49 (62.0\%) & $63(53.4 \%)$ & 0.455 \\
\hline $\begin{array}{l}\text { Intraoperative blood } \\
\text { loss, } \mathrm{mL}\end{array}$ & $\begin{array}{l}500 \\
(250-1300)\end{array}$ & $500(300-763)$ & 0.613 \\
\hline Operation time, $\min$ & $\begin{array}{l}300 \\
(192-411)\end{array}$ & $290(203-430)$ & 0.716 \\
\hline $\begin{array}{l}\text { Adjuvant chemo- and } \\
\text { radiotherapy }\end{array}$ & 31 (39.2\%) & 49 (41.5\%) & 0.749 \\
\hline
\end{tabular}

Abbreviation: AJCC, American Joint Committee on Cancer.

liver-directed therapy for recurrences, such as surgical resection, transarterial chemoembolization, and radiofrequency ablation, might improve the prognosis of patients with recurrent ICC. ${ }^{22-24}$ In addition, although the effect of adjuvant chemotherapy on the prognosis of ICC patients remains debatable, several studies have demonstrated potential survival benefits of adjuvant chemotherapy among "high-risk" patients with advanced tumor characteristics, such as nodal metastasis, advanced stages, or an inadequate margin. ${ }^{1,25,26}$ As such, patients with MiVI should similarly be considered for appropriate adjuvant therapy.
While DFS was comparable among patients with MiVI and MaVI, median OS was longer among patients with MiVI (39.0 vs 21.0 months). In fact, despite differences in DFS, OS of patients with MiVI was even comparable to the OS of patients who had NoVI (39.0 vs 45.0 months). While the reasons for these differences were undoubtedly multifactorial, these findings can be explained in part by the fact that patients with MiVI had a higher utilization of curative treatment of recurrences than patients with $\mathrm{MaVI}$ ( $17.7 \%$ vs $7.0 \%$ ). While data on the extent/burden of tumor recurrence were not available, the higher utilization of repeat curative-intent surgery among patients with MiVI strongly implied a less aggressive phenotype of intrahepatic-only recurrence among patients with MiVI vs MaVI. To this point, median OS after the first recurrence among patients with MiVI was more than double that of patients with MaVI (17.0 vs 8.0 months).

In examining survival, patients with MiVI had a comparable prognosis as patients with MaVI within the first 1 to 2 years after surgery yet diverged at 18 to 24 months. Previous work from our group had suggested that the timing of early vs late recurrence among patients with ICC could be defined empirically using a cutoff of about 2 years. ${ }^{6}$ In addition, among patients who did recur, the overwhelming majority recurred early. Similarly, in the present study, we were able to identify two prognostic cohorts among patients who had MiVI. In particular, there was a subset of patients who experienced early recurrence and death within the first 18 to 24 months after surgery. Interestingly, on multivariable analysis, factors associated with early mortality included multiple tumors (26.6\% vs $13.6 \% ; P=0.054)$, lymph node metastasis $(32.9 \%$ vs $16.0 \% ; P=0.024)$, and an $\mathrm{R} 1$ resection $(31.6 \%$ vs $13.6 \%$; $P=0.003)^{5,27-31}$ In contrast, among patients who were late survivors, the only factor associated with prognosis was lymph node metastasis, as patients with the nodal disease had a threefold increased risk of late death (HR 3.3; 95\% Cl 1.5-7.0; $P=0.002$ ). Lymph node status has previously been documented as one of the strongest prognostic factors associated with outcomes among patients with ICC. ${ }^{32-38}$ The present study highlights how lymph node status remained a strong indicator of prognosis even among patients with other risk factors such as MiVI. As such, routine lymphadenectomy to assess the nodal basin should be performed at the time of surgery to obtain important prognostic information, guide adjuvant therapy recommendations, as well as possibly prevent hilar nodal recurrence. ${ }^{34,39-42}$

Several limitations need to be considered when interpreting the present study. While the multicenter nature of the study undoubtedly increased sample size and analytical power, selection bias and variation in treatment strategies were possible. For example, patient selection for surgical resection has evolved over time and surgical approaches may have varied across the different centers. In addition, the diagnosis of MiVI was largely dependent on sample collection and histological examination. Therefore, it was possible that some variability in reporting of MiVI may have occurred, although this was likely low as the participating hospitals were major HPB centers with expertise in hepatopathology. 
TAB LE 5 Risk factors for early death ( $\leq 18$ months) of patients undergoing curative-intent resection for intrahepatic cholangiocarcinoma with microvascular invasion

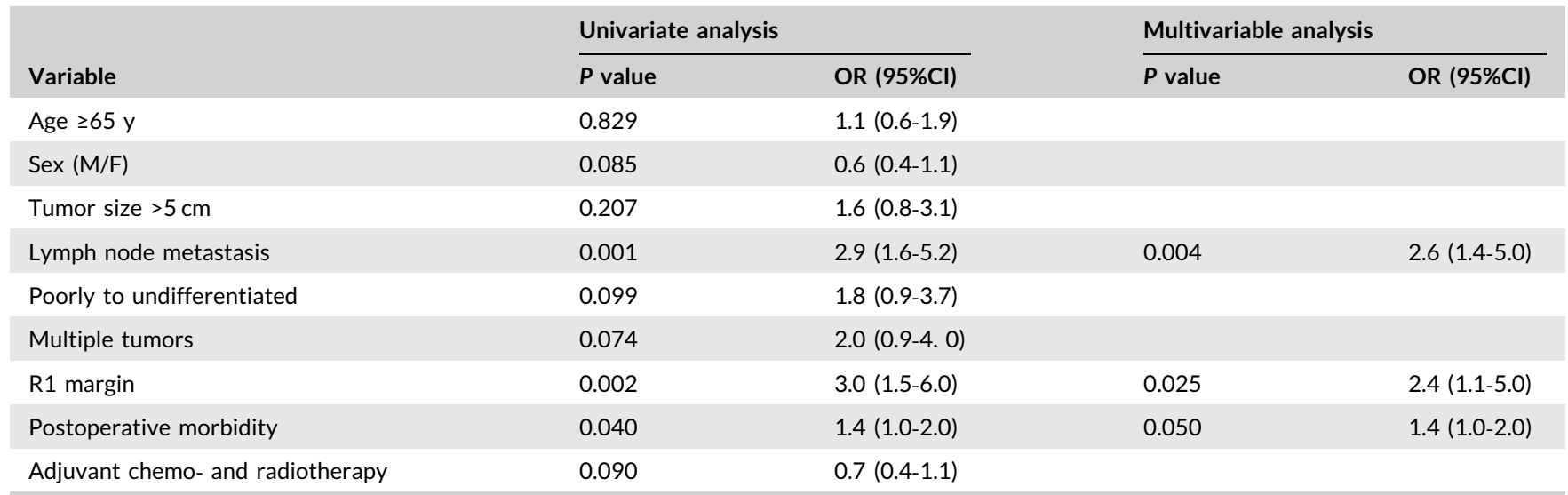

Abbreviations: $\mathrm{Cl}$, confidence intervals; OR, odds ratio.

TAB LE 6 Risk factors for the overall survival of patients with MiVI who survive longer than 18 months

\begin{tabular}{lll} 
& \multicolumn{2}{l}{ Univariate analysis } \\
\cline { 2 - 3 } Variable & P value & OR (95\%Cl) \\
\hline Age $\geq 65 \mathrm{y}$ & 0.812 & $1.1(0.5-2.3)$ \\
\hline Sex $(\mathrm{M} / \mathrm{F})$ & 0.357 & $0.7(0.3-1.5)$ \\
\hline Tumor size $>5 \mathrm{~cm}$ & 0.657 & $1.2(0.5-2.8)$ \\
\hline Lymph node metastasis & 0.002 & $3.3(1.5-7.0)$ \\
\hline Poorly to undifferentiated & 0.477 & $1.5(0.5-4.1)$ \\
\hline Multiple tumors & 0.443 & $1.5(0.5-4.5)$ \\
\hline R1 margin & 0.505 & $0.7(0.2-2.1)$ \\
\hline Postoperative morbidity & 0.700 & $0.9(0.6-1.5)$ \\
\hline Adjuvant chemo- and radiotherapy & 0.542 & $0.8(0.5-1.5)$ \\
\hline
\end{tabular}

Abbreviations: $\mathrm{Cl}$, confidence intervals; OR, odds ratio.

In conclusion, among patients undergoing curative resection for ICC, roughly one out of five patients had MiVI on histological examination, while one out of ten patients had the macrovascular invasion. MiVI was associated with advanced tumor characteristics, and thus a higher risk of tumor recurrence. Risk of early death among patients with MiVI was associated with tumor and surgical factors, while the risk of late death was largely impacted by lymph node status. MiVI status should be routinely documented in the pathology report of patients undergoing resection of ICC as these data have prognostic and possibly treatment-related implications.

\section{ACKNOWLEDGMENT}

The authors would like to acknowledge Dr. Xu-Feng Zhang, MD who also contributed to this manuscript.

\section{CONFLICTS OF INTEREST}

The authors declare that they have no conflicts of interest.

\section{ORCID}

Matthew Weiss (D) http://orcid.org/0000-0003-0553-248X

Timothy M. Pawlik (D) http://orcid.org/0000-0002-4828-8096

\section{REFERENCES}

1. Mavros MN, Economopoulos KP, Alexiou VG, Pawlik TM. Treatment and prognosis for patients with intrahepatic cholangiocarcinoma: systematic review and meta-analysis. JAMA Surg. 2014;149:565-574.

2. Aljiffry M, Abdulelah A, Walsh M, Peltekian K, Alwayn I, Molinari M. Evidence-based approach to cholangiocarcinoma: a systematic review of the current literature. J Am Coll Surg. 2009;208:134-147.

3. Amini N, Ejaz A, Spolverato G, Kim Y, Herman JM, Pawlik TM. Temporal trends in liver-directed therapy of patients with intrahepatic cholangiocarcinoma in the United States: a population-based analysis. J Surg Oncol. 2014;110:163-170.

4. Spolverato G, Kim Y, Alexandrescu S, et al. Management and outcomes of patients with recurrent intrahepatic cholangiocarcinoma following previous curative-intent surgical resection. Ann Surg Oncol. 2016;23:235-243.

5. Farges O, Fuks D, Boleslawski E, et al. Influence of surgical margins on outcome in patients with intrahepatic cholangiocarcinoma: a multicenter study by the AFC-IHCC-2009 study group. Ann Surg. 2011;254:824-829.

6. Zhang XF, Beal EW, Bagante F, et al. Early versus late recurrence of intrahepatic cholangiocarcinoma after resection with curative intent. Br J Surg. 2018;105:848-856.

7. Doussot A, Lim C, Gómez-Gavara C, et al. Multicentre study of the impact of morbidity on long-term survival following hepatectomy for intrahepatic cholangiocarcinoma. Br J Surg. 2016;103:1887-1894.

8. Sasaki K, Margonis GA, Andreatos N, et al. Preoperative risk score and prediction of long-term outcomes after hepatectomy for intrahepatic cholangiocarcinoma. J Am Coll Surg. 2018;226:393-403.

9. MB A: American Joint Committee on Cancer (2017) AJCC cancer staging manual Chicago, American Joint Committee on Cancer. Springer.

10. Rodríguez-Perálvarez M, Luong TV, Andreana L, Meyer T, Dhillon AP, Burroughs AK. A systematic review of microvascular invasion in hepatocellular carcinoma: diagnostic and prognostic variability. Ann Surg Oncol. 2013;20:325-339.

11. Onaca N, Davis GL, Jennings LW, Goldstein RM, Klintmalm GB. Improved results of transplantation for hepatocellular carcinoma: a report from the International Registry of Hepatic Tumors in Liver Transplantation. Liver Transpl. 2009;15:574-580. 
12. Mazzaferro V, Llovet JM, Miceli R, et al. Predicting survival after liver transplantation in patients with hepatocellular carcinoma beyond the Milan criteria: a retrospective, exploratory analysis. Lancet Oncol. 2009;10:35-43.

13. Wang CC, Iyer SG, Low JK, et al. Perioperative factors affecting long-term outcomes of 473 consecutive patients undergoing hepatectomy for hepatocellular carcinoma. Ann Surg Oncol. 2009;16:1832-1842.

14. Sumie S, Kuromatsu R, Okuda K, et al. Microvascular invasion in patients with hepatocellular carcinoma and its predictable clinicopathological factors. Ann Surg Oncol. 2008;15:1375-1382.

15. Roayaie S, Blume IN, Thung SN, et al. A system of classifying microvascular invasion to predict outcome after resection in patients with hepatocellular carcinoma. Gastroenterology. 2009;137:850-855.

16. Portolani N, Coniglio A, Ghidoni S, et al. Early and late recurrence after liver resection for hepatocellular carcinoma: prognostic and therapeutic implications. Ann Surg. 2006;243:229-235.

17. Pommergaard HC, Rostved AA, Adam R, et al. Vascular invasion and survival after liver transplantation for hepatocellular carcinoma: a study from the European Liver Transplant Registry. HPB (Oxford). 2018;20:768-775.

18. Zhang X, Li J, Shen F, Lau WY. Significance of presence of microvascular invasion in specimens obtained after surgical treatment of hepatocellular carcinoma. J Gastroenterol Hepatol. 2018;33:347-354.

19. Sun JJ, Wang K, Zhang CZ, et al. Postoperative adjuvant transcatheter arterial chemoembolization after RO hepatectomy improves outcomes of patients who have hepatocellular carcinoma with microvascular invasion. Ann Surg Oncol. 2016;23:1344-1351.

20. Ke-Wei L, Tian-Fu W, Xi L, et al. The effect of postoperative TACE on prognosis of HCC with microscopic venous invasion. Hepatogastroenterology. 2012;59:1944-1946.

21. Wang SN, Chuang SC, Lee KT. Efficacy of sorafenib as adjuvant therapy to prevent early recurrence of hepatocellular carcinoma after curative surgery: a pilot study. Hepatol Res. 2014;44:523-531.

22. Si A, Li J, Xing X, et al. Effectiveness of repeat hepatic resection for patients with recurrent intrahepatic cholangiocarcinoma: factors associated with long-term outcomes. Surgery. 2017;161:897-908.

23. Park HM, Yun SP, Lee EC, et al. Outcomes for patients with recurrent intrahepatic cholangiocarcinoma after surgery. Ann Surg Oncol. 2016;23:4392-4400.

24. Zhang SJ, Hu P, Wang N, et al. Thermal ablation versus repeated hepatic resection for recurrent intrahepatic cholangiocarcinoma. Ann Surg Oncol. 2013;20:3596-3602.

25. Miura JT, Johnston FM, Tsai S, et al. Chemotherapy for surgically resected intrahepatic cholangiocarcinoma. Ann Surg Oncol. 2015;22:3716-3723.

26. Reames BN, Bagante F, Ejaz A, et al. Impact of adjuvant chemotherapy on survival in patients with intrahepatic cholangiocarcinoma: a multi-institutional analysis. HPB. 2017;19:901-909.

27. $\mathrm{Li} \mathrm{M}, \mathrm{Bi} X$, Li Z, et al. Impaction of surgical margin status on the survival outcome after surgical resection of intrahepatic cholangiocarcinoma: a systematic review and meta-analysis. J Surg Res. 2016;203:163-173.

28. Uenishi T, Kubo S, Yamazaki $O$, et al. Indications for surgical treatment of intrahepatic cholangiocarcinoma with lymph node metastases. J Hepatobiliary Pancreat Surg. 2008;15:417-422.

29. Spolverato G, Yakoob MY, Kim Y, et al. The impact of surgical margin status on long-term outcome after resection for intrahepatic cholangiocarcinoma. Ann Surg Oncol. 2015;22:4020-4028.
30. Spolverato G, Yakoob MY, Kim Y, et al. Impact of complications on long-term survival after resection of intrahepatic cholangiocarcinoma. Cancer. 2015;121:2730-2739.

31. Ma KW, Cheung TT, She WH, et al. Major postoperative complications compromise oncological outcomes of patients with intrahepatic cholangiocarcinoma after curative resection-A 13-year cohort in a tertiary center. Asian J Surg. 2018.

32. Uchiyama $\mathrm{K}$, Yamamoto $\mathrm{M}$, Yamaue $\mathrm{H}$, et al. Impact of nodal involvement on surgical outcomes of intrahepatic cholangiocarcinoma: a multicenter analysis by the Study Group for Hepatic Surgery of the Japanese Society of Hepato-Biliary-Pancreatic Surgery. J Hepatobiliary Pancreat Sci. 2011;18:443-452.

33. Jutric Z, Johnston WC, Hoen HM, et al. Impact of lymph node status in patients with intrahepatic cholangiocarcinoma treated by major hepatectomy: a review of the National Cancer Database. HPB (Oxford). 2016;18:79-87.

34. Bagante F, Gani F, Spolverato G, et al. Intrahepatic cholangiocarcinoma: prognosis of patients who did not undergo lymphadenectomy. J Am Coll Surg. 2015;221:1031-1040. e1031-1034.

35. Bagante F, Spolverato G, Weiss M, et al. Assessment of the lymph node status in patients undergoing liver resection for intrahepatic cholangiocarcinoma: the New Eighth Edition AJCC Staging System. $J$ Gastrointest Surg. 2018;22:52-59.

36. Choi SB, Kim KS, Choi JY, et al. The prognosis and survival outcome of intrahepatic cholangiocarcinoma following surgical resection: association of lymph node metastasis and lymph node dissection with survival. Ann Surg Oncol. 2009;16:3048-3056.

37. Zhang XF, Chakedis J, Bagante $F$, et al. Trends in use of lymphadenectomy in surgery with curative intent for intrahepatic cholangiocarcinoma. Br J Surg. 2018;105:857-866.

38. Zhang XF, Chen Q, Kimbrough CW, et al. Lymphadenectomy for intrahepatic cholangiocarcinoma: has nodal evaluation been increasingly adopted by surgeons over time? A national database analysis. J Gastrointest Surg. 2018;22:668-675.

39. Zhang XF, Chakedis J, Baganate F, et al. Trends in utilization of lymphadenectomy in curative-intent surgery for intrahepatic cholangiocarcinoma: an international multi-institutional study. J Am Coll Surg. 2017. Under review.

40. Bagante F, Spolverato G, Weiss M, et al. Assessment of the lymph node status in patients undergoing liver resection for intrahepatic cholangiocarcinoma: the New Eighth Edition AJCC Staging System. J Gastrointest Surg. 2017;22:52-59.

41. Amini N, Ejaz A, Spolverato G, Maithel SK, Kim Y, Pawlik TM. Management of lymph nodes during resection of hepatocellular carcinoma and intrahepatic cholangiocarcinoma: a systematic review. J Gastrointest Surg. 2014;18:2136-2148.

42. Weber SM, Ribero D, O'Reilly EM, Kokudo N, Miyazaki M, Pawlik TM. Intrahepatic cholangiocarcinoma: expert consensus statement. HPB. 2015;17:669-680.

How to cite this article: Hu L-S, Weiss M, Popescu I, et al. Impact of microvascular invasion on clinical outcomes after curative-intent resection for intrahepatic cholangiocarcinoma. J Surg Oncol. 2019;119:21-29. https://doi.org/10.1002/jso.25305 